\title{
Éditorial : Les politiques françaises et canadiennes d'intégration à la lumière du " tournant civique »
}

The Editorial: French and Canadien Policies of Integration in the Light of the "civic turn"

Editorial: Las políticas francesas y canadienses de integración desde la perspectiva del «giro cívico»

Janie Pélabay, Elke Winter, Myriam Hachimi-Alaoui et Delphine Nakache

\section{(2) OpenEdition \\ Journals}

\section{Édition électronique}

URL : https://journals.openedition.org/remi/17015

DOI : 10.4000/remi. 17015

ISSN : $1777-5418$

\section{Éditeur}

Université de Poitiers

\section{Édition imprimée}

Date de publication : 31 décembre 2020

Pagination : 7-11

ISBN : 979-10-90426-68-9

ISSN : 0765-0752

\section{Référence électronique}

Janie Pélabay, Elke Winter, Myriam Hachimi-Alaoui et Delphine Nakache, «Éditorial : Les politiques françaises et canadiennes d'intégration à la lumière du « tournant civique » », Revue européenne des migrations internationales [En ligne], vol. 36 - n4 | 2020, mis en ligne le 01 janvier 2021, consulté le 15 avril 2022. URL : http://journals.openedition.org/remi/17015; DOI : https://doi.org/10.4000/remi. 17015 


\title{
Éditorial : Les politiques françaises et canadiennes d'intégration à la lumière du " tournant civique "
}

\author{
Janie Pélabay ${ }^{1}$, Elke Winter², \\ Myriam Hachimi-Alaoui ${ }^{3}$ et Delphine Nakache ${ }^{4}$
}

\begin{abstract}
Au cours des années 2000, les politiques d'immigration et de citoyenneté instaurées en Europe et en Amérique du Nord donnent lieu à la formulation d'un nouveau paradigme d'action publique, qualifié d' "intégration civique " (Joppke, 2007 ; Mouritsen, 2008). Dans les travaux qui leur sont consacrés, ces politiques sont présentées comme marquant un " tournant " (" civic turn »), visible dans la mise en place d'une série de dispositifs et de lois en matière d'entrée, de séjour, d'intégration des étranger-ère-s et d'acquisition de la nationalité. En font partie la multiplication de tests de citoyenneté, le renforcement des exigences linguistiques, le développement de formations civiques obligatoires, l'importance accordée aux cérémonies de citoyenneté dans le cadre de la naturalisation, la signature de chartes ou de contrats relatifs à l'intégration et à l'adhésion aux "valeurs " de la société d'installation.
\end{abstract}

Au-delà des discussions académiques sur la nature, l'ampleur et la portée d'un tel "tournant ", un consensus existe pour considérer que les reconfigurations à l'œuvre dans les politiques d' " intégration civique " consistent, d'une part, à imposer des contraintes plus fortes aux personnes migrantes désirant s'établir dans I'un des pays concernés et, d'autre part, à promouvoir une conception plus " épaisse " (" thick ") de la citoyenneté (Etzioni, 2011), où prime la dimension identitaire de l'appartenance à la communauté nationale.

\footnotetext{
1 Chargée de recherche FNSP, Sciences Po, Centre de recherches politiques (CEVIPOF), CNRS, 98 rue de I'Université, 75007 Paris, France ; janie.pelabay@sciencespo.fr 2 Full Professor of Sociology, University of Ottawa; 120 University Private (\#10059), Ottawa, Ontario, K1N 6N5, Canada; elke.winter@uottawa.ca

3 Maîtresse de conférences, Fellow à I'Institut Convergence Migration, Université le Havre Normandie, Laboratoire IDEES-Le Havre, 25 rue Ph. Lebon, 76000 Le Havre, France ; myriam.hachimi-alaoui@univ-lehavre.fr

4 Associate Professor, University of Ottawa, Faculty of Law, 57 Louis-Pasteur Private, Ottawa, Ontario, K1N6N5, Canada; dnakache@uottawa.ca

Ce numéro spécial n'aurait pas été possible sans le financement généreux du Fonds France Canada pour la Recherche (FFCR) pour le projet Être ou ne pas être (un bon) citoyen : The Role of Citizenship Acquisition for Immigrant Integration Trajectories in Canada and France (2017-2019) sous la direction de Myriam Hachimi-Alaoui et d'Elke Winter, et du Canada Program de I'Université Harvard lorsqu'Elke Winter était titulaire de la Chaire William Lyon Mackenzie King en études canadiennes (2019-2021). Un grand merci également aux évaluateurs et évaluatrices externes ainsi qu'à toute l'équipe de la REMI.
} 
Alors que l'intégration des migrant.e-s est au cœur du débat public, le présent dossier propose $d^{\prime}$ analyser les politiques $d^{\prime}$ ' intégration civique " en se centrant sur deux cas d'étude : la France et le Canada. À l'aide d'analyses qui allient recherches empiriques et approches théoriques, et en s'appuyant sur un vaste ensemble de données, il s'agit de comprendre si les reconfigurations mises au compte d'un "tournant civique " sont en cours dans les deux pays, et quelles sont leurs éventuelles incidences pour les migrant.e.s, en termes d'inclusion/ exclusion, et pour la société d'installation, en termes de réaffirmation de l'identité nationale. Pour ce faire, ce dossier de la REMI La citoyenneté au temps de "I'intégration civique " : regards croisés France/Canada suit une démarche résolument interdisciplinaire, en combinant et croisant sociologie, anthropologie, droit, philosophie et science politique, non seulement au sein du dossier thématique dans sa globalité, mais aussi dans plusieurs des articles.

Analyser les politiques d'intégration en France et au Canada à travers le prisme du " tournant civique " permet, d'une part, d'en renouveler l'étude dans ces deux contextes nationaux en leur appliquant une grille de lecture peu utilisée jusqu'à présent. D'autre part, ces deux cas d'analyse permettent d'élargir la littérature sur le "tournant civique ", principalement composée d'études sur les Pays-Bas, le Royaume-Uni, I'Allemagne, les pays scandinaves ainsi que sur les États-Unis (Bonjour, 2018 ; Meer et al., 2015 ; Borevi et al., 2017 ; Goodman, 2020).

Les articles réunis contribuent ainsi aux trois débats que Joppke (2017) identifie comme étant au centre de la littérature sur les politiques $d^{\prime}$ ' intégration civique ". Premièrement, ces politiques opèrent-elles une convergence ou, au contraire, creusent-elles l'écart entre " modèles nationaux " ? Deuxièmement, marquentelles un recul vis-à-vis des politiques d'orientation multiculturaliste, ou se superposent-elles à ces dernières ? Troisièmement, soutiennent-elles - comme le pense Joppke (2010) - une vision " libérale " de la citoyenneté, tournée vers les droits fondamentaux et les libertés individuelles, ou signalent-elles, au contraire, le retour de "l'assimilation culturelle " (Duyvendak et al., 2016) ?

La question d'un " tournant civique " en France et au Canada a pour arrièreplan les différences posées entre deux " modèles" - le premier qualifié de " républicain ", le second de " multiculturaliste " - se prévalant d'orientations idéologiques divergentes dont il faut tenir compte.

S'agissant de la France, tester I'hypothèse d'un " tournant civique " appelle d'emblée un certain nombre de remarques. Notons d'abord que ce n'est pas, ou pas principalement, en ces termes que se déroule le débat, tant public qu'académique, sur l'entrée des étranger-ère-s dans la communauté nationale. Au début des années 2000 comme aujourd'hui, le débat porte moins sur le " modèle français d'intégration " que sur la possibilité et la désirabilité de réaménagements visant à le rendre plus hospitalier aux différences culturelles. Et même lorsque les pouvoirs publics revendiquent l'amorce de " politiques de la diversité ", c'est en prenant soin de les distinguer du "multiculturalisme anglo-saxon " qui tient un rôle de " repoussoir " dans le débat français. Ensuite, les termes mêmes de ce débat, comparés à ceux employés dans la littérature sur le " tournant civique ", manifestent certaines spécificités et dissonances sémantiques qui peuvent compliquer l'application de cette grille d'analyse au cas de la France. II en va ainsi du "libéralisme ", largement associé au néolibéralisme économique, alors que dans les travaux sur la citoyenneté, ce terme porte une 
conception de la justice alliant respect des droits fondamentaux et recherche de justice sociale. Absolument central dans le débat français, le mot " communautarisme " englobe un ensemble de phénomènes, réels ou fantasmés, contre lesquels n'ont de cesse d'alerter ceux-là mêmes dont la conception "épaisse " de la citoyenneté se rapproche pourtant le plus de celle que la littérature discute sous le vocable de pensée "communautarienne "(Lacroix, 2007). Enfin, la question, soulevée dans la littérature sur l' "intégration civique ", d'un abandon des politiques multiculturalistes prend, dans le cas français, une allure singulière, sinon paradoxale : la validation de "l'échec du multiculturalisme " par le président Nicolas Sarkozy en 2011 ne saurait indiquer un quelconque " tournant " puisque la France n'a jamais adopté de politiques rentrant dans cette catégorie. Plus encore, en terre "républicaine ", cet échec proclamé par Angela Merkel, puis David Cameron est tenu pour la preuve tangible de la supériorité du "modèle français ", présenté comme plaçant les attachements " civiques " à l'abri et au-dessus des appartenances dites " ethniques ". Ce sont là des éléments qui demandent à être pris en compte pour instruire le dossier d'un " tournant civique " à la française, a fortiori quand l'éventuel virage consisterait à adopter une orientation " civique " d'avec laquelle les pouvoirs publics français n'ont jamais déclaré vouloir rompre.

Dans le débat public sur l'intégration, le Canada est également considéré - à tort ou à raison - comme possédant un positionnement idéologique et politique relativement stable. Dans ce cas, c'est le modèle "multiculturaliste " qui constitue l'arrière-plan à partir duquel se pose la question d'un éventuel "tournant civique ", soutenant une conception plus "épaisse " de la citoyenneté. Dans une certaine mesure, I'approche du Canada en matière d'intégration a depuis longtemps été "civique " dans le sens où la politique du multiculturalisme encourage non seulement la valorisation de la diversité culturelle, mais aussi l'intégration et la constitution d'une identité commune dans une société majoritaire. C'est la même chose pour l'interculturalisme québécois qui - plus encore que le multiculturalisme dans le reste du Canada - met l'accent sur un noyau culturel (la culture canadienne-française notamment) et demeure étroitement lié à la "francisation" des nouveaux.elles arrivant.e.s. Dans les deux cas, on est loin d'une politique encourageant l'existence de " sociétés parallèles ". Au contraire, le multiculturalisme canadien se veut un moyen de renforcer l'identité nationale commune. À la différence de l’Europe où le " tournant civique " est étroitement lié à la mise en œuvre de tests, de cérémonies et de serments de citoyenneté, ces dispositifs ne sont pas nouveaux au Canada. Ils sont mis en place dès 1947 - avec l'entrée en vigueur de la première loi sur la citoyenneté canadienne - et constituent dès cette époque des exigences à respecter pour devenir citoyen-ne canadien.ne. Néanmoins, à partir des années 2000, un changement important peut être noté au niveau de la politique fédérale. Le gouvernement conservateur de Stephen Harper (2006-2015) s'inspire du prétendu " backlash " - réactions de rejet - contre le multiculturalisme en Europe pour redéfinir le Canada en termes résolument plus conservateurs et nationalistes. La visibilité et la portée du multiculturalisme sont réduites, la politique d'immigration est réorganisée selon des critères économiques et la citoyenneté devient plus difficile à obtenir et plus facile à perdre. Ces changements reposent sur une implication plus forte de l'État dans les politiques d'intégration tout en mettant l'accent sur la responsabilité individuelle des migrant.e.s. Ils visent, selon le gouvernement en place, à renforcer les valeurs et l'économie canadiennes que la globalisation rendrait plus vulnérables. 
Malgré les singularités propres à ces deux contextes, l'analyse des mesures à l'égard des migrant.e.s développées depuis les années 2000 en France et au Canada fait apparaître des reconfigurations de l'action publique correspondant au paradigme de l'" intégration civique ".

En ce qui concerne la France, I'article d'Émilien Fargues sur la condition $d^{\prime}$ " insertion professionnelle " et, en particulier, l'exigence de " performance économique " dans les procédures de naturalisation met en évidence, sur la longue durée, l'existence d'un alliage, a priori paradoxal, entre la dimension communautarienne des politiques d' " intégration civique " et une orientation néolibérale. Analysant les contrats d'intégration de 2003 à aujourd'hui, Myriam Hachimi-Alaoui et Janie Pélabay montrent que la " condition d'intégration républicaine ", adossée à l'obligation faite aux migrant·e-s de se conformer aux " valeurs de la République ", indique une moralisation et une culturalisation de la citoyenneté. C'est en s'intéressant aux pratiques locales d' "intégration participative " mises en place dans une association de la banlieue parisienne s'adressant aux femmes migrantes que Linda Haapajärvi documente cette tendance double à culturaliser et à moraliser la citoyenneté, notamment au travers d'une injonction d'émancipation et d'épanouissement individuel.

Le cas du Canada est, quant à lui, traité à travers deux articles et une note de recherche. Delphine Nakache, Jennifer Stone et Elke Winter analysent la récente baisse du taux de naturalisation au Canada. Elles montrent que l'accent mis sur la sélection économique et la responsabilisation des individus en matière $d^{\prime}$ " intégration " nuisent à ceux - et surtout à celles - qui s'installent au Canada par des voies non économiques, en les privant d'accès à la citoyenneté. Lois Harder examine les débats sur le " tourisme de maternité " et la manière dont ils révèlent des préjugés sur ce qu'est une citoyenneté jugée " convenable ". En prenant pour objet d'étude le nouveau " test des valeurs québécoises ", obligatoire depuis janvier 2020 pour les migrant·e-s économiques qui désirent s'installer dans la province, Emily Laxer analyse, dans sa note de recherche, le cœur même des outils politiques privilégiés par les promoteurs de l'« intégration civique ".

Comme le souligne le texte conclusif de Saskia Bonjour en apportant un éclairage " postcolonial " à ce dossier, les analyses qu'il réunit révèlent, chacune à leur manière, que les politiques développées en France et au Canada renferment effectivement les principaux traits du " tournant civique " tels que dépeints par Mouritsen et al. (2019 : 601), à savoir : la responsabilisation des individus dans la " réussite " de " leur " intégration, l'intervention de l'État sur le plan des valeurs et de l'identité personnelles, l'usage des dispositifs d'intégration pour écarter les " indésirables ", la culturalisation de la "bonne citoyenneté ". L'un des enseignements forts de l'étude croisée des cas français et canadien est que c'est moins sous forme de rupture que d'approfondissement de tendances déjà à l'œuvre que se signale, dans les deux contextes, la présence de politiques $\mathrm{d}^{\prime}$ " intégration civique ". Au-delà de ce constat, la mise en commun des analyses consacrées à la France et au Canada fait surgir une question transversale sur la nouveauté et la force d'un " tournant néolibéral " qui viendrait redoubler ces politiques et intensifier leurs effets sur le traçage de frontières toujours plus " épaisses " entre " nous " et les " autres". 


\section{Références bibliographiques}

Bonjour Saskia (2018) The Role of the State and the Image of Migrants: Debating Dutch Civic Integration Policies, in Andrea Rea, Emmanuelle Bribosia, Isabelle Rorive and Djordje Sredanovic Eds., Governing Diversity: Migrant Integration and Multiculturalism in North America and Europe, Bruxelles, Editions de I'Université de Bruxelles, pp. 203-217.

Borevi Karin, Jensen Kristian K. and Mouritsen Per (2017) The civic turn of immigrant integration policies in the Scandinavian welfare states, Comparative Migration Studies, 5 (9), [online]. URL: https://doi.org/10.1186/s40878-017-0052-4

Duyvendak Jan Willem, Geschiere Peter and Tonkens Evelien (Eds.) (2016) The Culturalization of Citizenship: Belonging and Polarization in a Globalizing World, London, Palgrave Macmillan.

Etzioni Amitia (2011) Citizenship in a communitarian perspective, Ethnicities, 11 (3), pp. 336-349.

Goodman Sara Wallace (2020) "Good American citizens": a text-as-data analysis of citizenship manuals for immigrants, 1921-1996, Journal of Ethnic and Migration Studies, pp. 1-24, [online]. URL: https://doi.org/10.1080/1369183X.2020.1785852

Joppke Christian (2017) Civic integration in Western Europe: three debates, West European Politics, 40 (6), pp. 1153-1176.

Joppke Christian (2010) Citizenship and Immigration, Cambridge, Cambridge University Press.

Joppke Christian (2007) Beyond national models: Civic integration policies for immigrants in Western Europe, West European Politics, 30 (1), pp. 16-22.

Lacroix Justine (2007) Communautarisme et pluralisme dans le débat français. Essai d'élucidation, Éthique publique, 9 (1), pp. 50-55.

Meer Nasar, Mouritsen Per, Faas Daniel and de Witte Nynke (2015) Examining "Postmulticultural" and Civic Turns in the Netherlands, Britain, Germany, and Denmark, American Behavioral Scientist, 59 (6), pp. 702-726.

Mouritsen Per (2008) Political Responses to Cultural Conflict: Reflections on the Ambiguities of the Civic Turn, in Per Mouritsen and Knud Erik Jørgensen Eds., Constituting Communities: Political Solutions to Cultural Conflict, Basingstoke, Palgrave Macmillan, pp. 1-30.

Mouritsen Per, Jensen Kristian K. and Larin Stephen J. (2019) Introduction: Theorizing the civic turn in European integration policies, Ethnicities, 19 (4), pp. 595-613. 